\title{
Leprosy exposure, infection and disease: a 25-year surveillance study of leprosy patient contacts
}

\author{
Euzenir Nunes Sarno ${ }^{1 /+}$, Nadia Cristina Duppre ${ }^{1}$, Anna Maria Sales ${ }^{1}$, \\ Mariana Andréa Hacker', José Augusto Nery', Haroldo José de Matos² \\ 'Laboratório de Hansenologia, Instituto Oswaldo Cruz-Fiocruz, Rio de Janeiro, RJ, Brasil \\ ${ }^{2}$ Setor de Atendimento Médico Unificado, Instituto Evandro Chagas, Belém, PA, Brasil
}

\begin{abstract}
Contact surveillance is a valuable strategy for controlling leprosy. A dynamic cohort study of leprosy contacts was initiated in 1987 at Oswaldo Cruz Foundation. The objective of this work was to review the data on the major risk factors leading up to the infectious stage of the disease, estimate incidence rates of leprosy in the cohort and characterise the risk factors for the disease among the contacts under surveillance. The incidence rate of leprosy among contacts of leprosy patients was estimated at 0.01694 cases per person-year in the first five years of followup. The following factors were associated with acquiring the disease: (i) not receiving the BCG vaccine, (ii) a negative Mitsuda reaction and (iii) contact with a patient with a multibacillary clinical form of leprosy. The contacts of index patients who had high bacilloscopic index scores $>1$ were at especially high risk of infection. The following factors were associated with infection, which was defined as a seropositive reaction for anti-phenolic glicolipid-1 IgM: (i) young age (<20 years), (ii) a low measured Mitsuda reaction $(<5 \mathrm{~mm})$ and (iii) contact with an index patient who had a high bacilloscopic index. BCG vaccination and re-vaccination were shown to be protective among household contacts. The main conclusions of this study indicate an urgent need for additional leprosy control strategies in areas with a high incidence of the disease.
\end{abstract}

Key words: leprosy - contact surveillance - epidemiology - PGL-1

The leprosy elimination campaign sponsored by the World Health Organization (WHO) has successfully reduced the prevalence rate of the disease to less than one case per 10,000 inhabitants worldwide, but the number of new cases in endemic countries has remained stable or increased (Richardus \& Habbema 2007). In Brazil, for example, the elimination campaign has had mixed results. Leprosy continues to be a serious public health concern throughout the country, especially in the North and Central-West regions.

The high concentration of new cases among household contacts of leprosy patients has been demonstrated in numerous international studies (Fischer et al. 2010, Sales et al. 2011). Brazilian studies have also highlighted the elevated risk factors associated with these close contacts (Goulart et al. 2008, Sales et al. 2011).

In light of the elimination program sponsored by WHO, some studies have emphasised the need to identify index patients and treat them as a priority, especially in areas with low incidence rates, as discussed by Shen et al. (2008). However, some studies point to the concern that some countries may interrupt contact tracing and other initiatives, increasing the number of infectious patients (Fischer et al. 2011).

+Corresponding author: euzenir@fiocruz.br

Received 5 April 2012

Accepted 25 July 2012
In 1987, researchers began a long-term cohort study of individuals who have come into contact with leprosy patients diagnosed at the Souza Araújo Ambulatory, a regional and national centre for leprosy diagnosis and research headquartered at the Oswaldo Cruz Foundation (Fiocruz) in the city of Rio de Janeiro, which operates under the auspices of the Brazilian Ministry of Health.

The objective of the present review was to reflect on the experiences of this cohort of contacts over the past 25 years and compare the original data from this cohort (published and unpublished) with the data published in the literature to better understand how leprosy infection evolves into leprosy disease as a result of close proximity to leprosy patients.

From exposure to infection - Fully understanding leprosy infection is one of the most important and challenging aspects of investigating the epidemiology of the disease. Could a state of infection indicate subclinical disease? Could infected contacts be important links in the chain of transmission of Mycobacterium leprae? These questions, first posed by Noordeen in 1993, have remained largely unanswered.

Overall, immunological studies of leprosy have emphasised the central role played by the $M$. leprae-macrophage interaction in the pathogenesis of leprosy disease, prioritising experiments with interferon-gamma in the patients and contacts in the Fiocruz cohort mentioned above (Sampaio et al. 1991). However, while new diagnostic tests based on private peptides are being developed in the same Fiocruz laboratory (Bobosha et al. 2012), most authors agree that seropositivity of class IgM antiphenolic glicolipid (PGL)-1 antibodies indicates the pres- 
ence of a Hansen bacillus infection (van Beers et al. 1994, Moura et al. 2008). Moura presents a systematic review of seropositivity as an important instrument in controlling dissemination of the disease.

Therefore, in addition to studying household contacts, there are sero-epidemiological inquiries currently underway to characterise seropositivity for anti-PGL-1 (Moura et al. 2008, Barreto et al. 2011), specifically focused on identifying the infection in school-age populations within endemic areas. The use of rapid techniques, such as the maximum-likelihood-flow, has made these field studies possible.

After conducting surveillance for 10 years on a cohort of leprosy contacts in French Polynesia, Chanteau et al. (1993) concluded that the risk of developing leprosy was not significantly different when comparing seropositive and seronegative contacts ( $2 \%$ vs. $1 \%, p=0.2)$, indicating a low positive predictive value for the test. The authors demonstrated that detecting IgM anti-PGL-1 is not an effective tool for diagnosing leprosy in its earliest stages within a high-risk population in an endemic area.

Using data from the Fiocruz cohort of contacts, Saad et al. (1990) showed an association between anti-PGL-1 in contacts and patients using an ELISA technique for detecting anti-PGL-1 antibodies in serum using a nitrocellulose stick (dipstick). By studying healthy leprosy contacts who did not develop the disease by the end of the study, the results of this serology offered a new perspective on leprosy infection and disease. This study demonstrated that a state of infection does not necessarily lead to a state of disease, especially when the microorganism involved displays low pathogenicity, such as with M. leprae. Another hypothesis, in accordance with the definition presented by Fine (1982) that characterised the epidemiology of leprosy as a prototype of a slow infection, is that the disease is the result of a process that occurs in multiple stages. Chronic diseases, such as neoplasms, offer a typical example of this process, in which the infection is the first stage of the interaction between the microorganism and host. Additional factors are required to evolve to the next stage (i.e., progress to the state of disease or return to a previous stage if the infectious agent is eliminated or neutralised).

The objective of studying the cohort of leprosy contacts at the Fiocruz Souza Araújo Ambulatory beginning in 1987 was to investigate the factors associated with seropositivity among the healthy contacts. These contacts showed no evidence of disease when their index patient was diagnosed and no signs of the disease during reexamination. In addition, no alterations in the state of disease were reported while the researchers attempted to characterise the risk factors of infection (infection defined by IgM anti-PGL-1 seropositivity). It appears clear that any contact seropositivity that did not evolve into a state of disease (at least during the observation period) corroborates the idea that leprosy infection is only one stage in the interaction between M. leprae and the host. It is also useful to identify the specific factors associated with the infection because this knowledge may assist in developing new strategies for controlling leprosy in its earliest stages, in contrast with the aforementioned conclusion of Chanteau et al. (1993).
The results (Matos et al. 1999) showed that BCG vaccination does not appear to be significantly associated with seropositivity in healthy leprosy contacts in the studied cohort. These results are interesting because they show that there are more infected individuals among the vaccinated subjects than the non-vaccinated subjects [harzard natio $(\mathrm{HR})=1.348$ ]. Thus, BCG vaccination does not appear to actually prevent leprosy infection. Instead, BCG is associated with PGL-1 seropositivity. Whether this effect is due to the control of M. leprae spread by granuloma formation or another biological mechanism is still a matter of speculation. In any case, it would be interesting to study the effect of BCG vaccination on the evolution of PGL-1 levels in contacts.

The results also highlight the importance of adequately characterising the level of exposure among contacts, recognising the capacity of primary cases to eliminate the bacillus because the primary cases are the principle, if not the only, source of infection. Most epidemiological studies utilise the clinical classification system to indicate the infectiousness of the primary case. Thus, the most infectious cases would be categorised as the lepromatous leprosy and borderline lepromatous clinical forms, in which the patient does not seem to present a typical cellular immune response against $M$. leprae. The less infectious cases, however, are categorised as the indeterminate clinical form (the most common first clinical form of the disease), borderline tuberculoid form and tuberculoid tuberculoid form (the polar form, in which a response is clear).

For the purpose of treatment, the WHO has adopted a summary classification to describe the multibacillary (MB) and paucibacillary (PB) forms. This classification could have been used as an indirect measure of exposure in the model proposed for the seropositivity study, as a proxy for the duration of cohabitation between the contact and his or her leprosy patient. Nevertheless, during analysis, this variable was not considered as important as the bacillary index (BI) (model not presented in the results), which is a direct measure of the bacillary load calculated after identifying the alcohol-acid resistant bacilli in the lymph nodes at six sites in the body with an optical microscope. The BI is then transformed into a logarithmic scale as proposed by Ridley and Jopling (1962). What the penalised Cox model appears to confirm is that this measure is directly associated with anti-PGL-1 seropositivity and consequently, with infection, particularly concerning $\mathrm{BI}$ value 3 . Thus, when $\mathrm{BI}$ is used, additional information impacts the data, as the clinical form of the disease provides a measure of exposure to the Hansen bacillus. Recent analyses have considered the sum of all positive BIs, including the index patient and co-prevalent contacts and confirmed the role of infectivity in the incidence of leprosy cases among contacts (NC Duppre et al., unpublished observations).

Another important factor associated with IgM antiPGL-1 seropositivity was the age of the contact. It was believed that age might be closely associated with the length of time that the individual was directly or indirectly exposed to $M$. leprae. However, as seen below, no statistically significant association between the duration of coexistence and presence of anti-PGL-1 antibodies could be discerned from the contacts studied. On the 
contrary, an inverse association between age and seropositivity was found. Thus, using the Cox model, the younger contacts had a higher probability of seropositivity than the older contacts. Accordingly, there is a clear linear relationship that shows that increased risk is associated with younger age, especially for those under 20. It is astounding that these data corroborate the opinion expressed by Ridley and Jopling (1966) that leprosy infection occurs during infancy (Fig. 1).

The Mitsuda reaction, introduced in 1919 by Kensuke Mitsuda, is an intradermal reaction in response to inoculation with lepromin that was initially prepared by the investigators. This reaction contained a mixture of antigens obtained from patient lesions. Lepromin is currently obtained from infected armadillos and its preparation is standardised. Derivatives are also obtained from the suspension of bacilli and protein derivatives. The Mitsuda reaction is evaluated between 21-28 days after inoculation, when the maximum diameter of induration at the injection site is measured. Forty-eight hours after inoculation, an alternative reading is performed. This reading is known as the Fernandez reaction, after José Fernandez, an Argentinian who introduced this variation in 1940 and demonstrated a correlation between the two readings. However, some authors (Rodriguez \& Orozco 1996) interpret these two readings in different manners. The Fernandez reaction could be a delayed response showing hypersensitivity to $M$. leprae and potentially other mycobacteria, whereas the Mitsuda reaction is classically interpreted as a measurement of individual resistance and thus, would have prognostic value. Nevertheless, several authors (Rodriguez \& Orozco 1996) have suggested that the Mitsuda reaction could also indicate infection by acting as a diagnostic tool for infection rather than as a diagnostic tool for disease. Traditionally, the result of the Mitsuda test is reported as positive or negative based on the maximum diameter of induration. Measurements of induration that are $5.0 \mathrm{~mm}$ or greater (tuberculum) are considered positive readings according to the standard of the Brazilian Ministry of Health. In the penalised Cox model (Matos et al. 1999), however, the co-variable
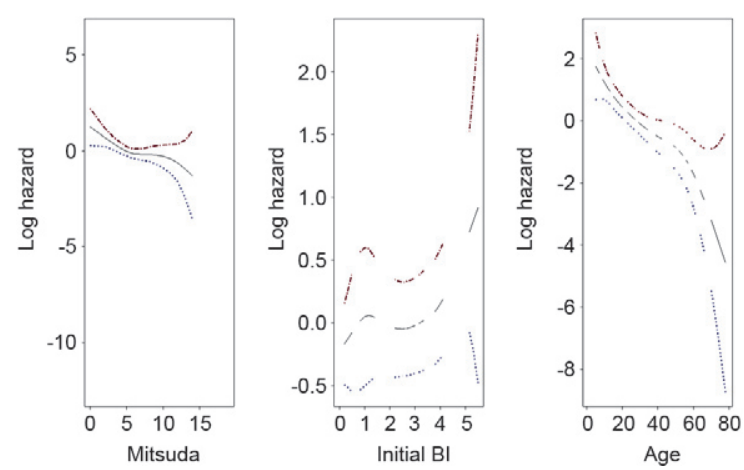

Fig. 1: representation of the adjustment of the terms "spline" for each one of the continual co-variables introduced in the penalized Cox model. The log hazards for seropositivity in function of the values of the co-variables are presented. BI: bacillary index. for the Mitsuda reaction is treated as a continuous variable. A "spline" function of the Mitsuda reading plotted against the individual values of the readings reveals that the contacts who have lower Mitsuda measurements have a greater probability of seropositivity and this probability diminishes as the value of the Mitsuda test increases, especially for values of $5.0 \mathrm{~mm}$ and greater. Thus, the seropositivity test for IgM anti-PGL-1 can be considered a marker of infection with M. leprae, especially in individuals with negative Mitsuda reactions.

Transmission models for infectious diseases have primarily studied the role of household contacts in disseminating leprosy (Richardus \& Habbena 2007). These models are based on the assumption that the rate of contact between a pair of individuals who live in the same household tends to be much greater than the contact between individuals who live in different households. These models have been very useful in formulating vaccination strategies for highly infectious diseases (i.e., diseases assumed to infect all members of a population if one member of the population becomes infected). In this manner, individuals residing in the same household would form an analytical unit distinct from the individuals dwelling in another household. In the present database, the leprosy patient and his/her contacts are aggregated into one unit and considered a family because they are living under the same roof, although they may not actually have any family ties. In the epidemiology of leprosy, there are little data concerning the incidence rate of leprosy disease in certain families. Although household contacts have a greater risk of developing leprosy than non-household contacts, Ranade and Joshi (1995) analysed leprosy incidence data in a hyper-endemic district in India and found that families as units had a risk similar to the risk of disease development among existing household contacts and additional cases. Alternatively, at least theoretically, belonging to a certain family could introduce an additional source of variability or increase in risk over time, which would be important to consider when applying a multivariate model, such as the Cox model.

For this reason, the random effects term, or frailty term, was introduced in the penalised Cox model. Still, although the introduction of the random effects term has been considered significant $(p=0.0002)$ in the adjustment of the model, the parameter estimates for the co-variables suffered very small variations. This result suggests that the family effect (present or not) did not significantly modify the HR estimates of the analysed variables. This appears to align with the empirical observations made by Ranade and Joshi (1995), at least in terms of the variables studied.

From the data presented thus far, it can be concluded that finding a relatively high prevalence of seropositivity for IgM anti-PGL-1 in healthy leprosy contacts $(20.8 \%)$ is significant. This relatively high rate contrasts with the relatively low incidence rates among contacts in this cohort and other groups that have been studied, which vary between $2-8 \%$ per person-year (Chanteau et al. 1993, Matos et al. 1999). However, this contrast supports the multi-stage $M$. leprae-host interaction model and the data provided by Chanteau et al. (1993), which 
indicated a low positive predictive value for IgM antiPGL-1 seropositivity.

In short, the model presented (Fig. 1) is a generalised linear model adjusted with cubic "splines" and the variables associated with seropositivity for PGL-1 ( $p<0.05$ using the Wald test) are age, Mitsuda reaction and bacilloscopic index.

It can be inferred that in many seropositive (i.e., infected) contacts, the infection does not evolve to disease. These contacts remain infected for an indefinite period of time, return to a previous stage, possibly harbouring $M$. leprae in macrophages, or may eliminate the bacteria altogether.

Finally, because there are rapid and cost-effective detection methods now available, it is both practical and useful to test for anti-PGL-1 antibodies. Especially in the high-incidence and endemic areas of leprosy, this test is an important tool for any contact surveillance program, offering a means of disease control and serving as an important complement to other elimination strategies. Similarly, there is enormous potential for the use of diagnostic tests based on M. leprae-specific peptides. As previously published results (Bobosha et al. 2012) show that these tests, apart from characterising contacts, present a much more fine-tuned capacity to detect exposure levels (i.e., infection) among both exposed and non-exposed populations, these studies also represent an important advance in the search for an effective vaccine against leprosy.

From the contact to disease - Studies on the risk of illness in cohorts of leprosy contacts that emphasise the important role played by household contacts in the epidemiology of leprosy have emerged in the last decade. These studies have drawn particular attention to the strategic possibilities that contact surveillance offers control and elimination of the disease, which is the basic objective of the WHO (1998).

Data from the leprosy contact cohort at Fiocruz have produced at least five different publications concerning the risk of illness among contacts (Alvim 1993, Matos et al. 1999, Duppre et al. 2008, Sales et al. 2011). Furthermore, a variety of studies have tested various genetic polymorphisms as susceptibility factors for leprosy among this same cohort of contacts (Vanderborght et al. 2004).

Having a genetic relationship increases the risk of acquiring leprosy among otherwise healthy household contacts. Moet et al. (2006) have shown that physical distance and genetic distance were independently associated with the risk of acquiring leprosy for patient contacts. Other studies have corroborated these findings (Abel \& Demenais 1988, Feitosa 1995, Lazaro et al. 2010, Feenstra et al.2012) and suggested a genetic susceptibility mechanism. Nevertheless, this review is primarily concerned with modifiable factors that could be used to improve surveillance.

Fig. 2 shows the leprosy incidence rate among contacts gleaned from data collected in the first 10 -year period of the study. The risk of illness is greatest in the first year after the diagnosis of the index patient. In this study (Matos et al. 1999), the overall incidence rate was 16.94 per 1,000 person-years of follow-up between 1987-1991. The calculation of person-years is based on the length of the contact's coexistence with the index patient, calculated from the initial detection of the primary case until the first to occur between the end of his/her active disease period or the healthy contact ceases to inhabit the same household. Some practical difficulties occur when applying this concept. First, it is difficult to establish with certainty the exact time period that the disease was active. Second, precise estimates of the time frame that an individual lived in a certain household were rare. Finally, an additional difficulty lies in determining the end of the risk period for the contact after the primary case has initiated treatment. Even when using the current multi-drug treatment authorised by the WHO, there is no consensus about when an individual can be considered cured of the disease.

Therefore, it is not surprising that the data on the annual incidence rates among contacts are lower in the later years of follow-up. Once the individual is treated, the probability of illness among his/her household contacts tends to fall. Nevertheless, secondary cases continue to occur, although they occur much more rarely. This result may be due to the long incubation period of the leprosy bacillus subsequent to or combined with lengthy periods of exposure. It is important to keep in mind that an individual may be infected, but show no signs of the disease. Nordeen (1993) characterised these individuals as carriers in their review of the epidemiology of leprosy. A second interpretation is that the source of infection for these secondary cases (e.g., cases diagnosed after more than 2 years of follow-up) could be another individual, either someone outside the family or an asymptomatic carrier of leprosy in the family. The possibility that asymptomatic carriers of leprosy actually exist and that these carriers could be the source of infection for a household contact cannot be easily discarded, due to the lack of evidence. In addition, SIMCOLEP modelling appears to indicate that it is essential to investigate these possibilities so that additional control measures could be developed.

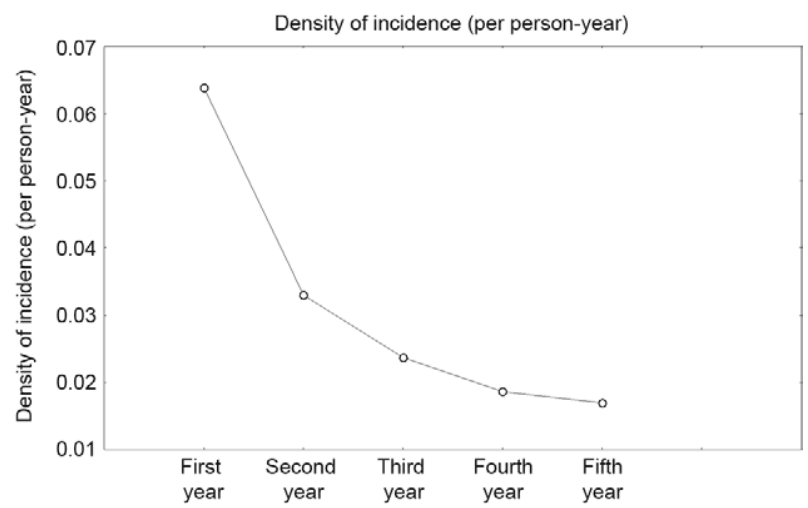

Fig. 2: density of incidence in persons-year of follow-up. The values plotted for the rate of incidence are the values of the rate of accumulated incidence, i.e., the first value plotted was estimated up to the end of the first year of follow-up, the second up to the end of the second year, the third up to the end of the third, the fourth up to the end of the fourth and the fifth up to last date between the fourth and the fifth year of accompanying. 
Many people consider contact surveillance to be unimportant in endemic areas, but our results on leprosy incidence rates among household contacts demonstrate that it is important. In 1987, the average incidence rate of leprosy in Brazil was 1.42/1,000 individuals. Compared with this incidence rate among the general population, the incidence rate among the contacts in our study was more than 12-fold higher, indicating a resistant ratio (RR) of 12. Chanteau et al. (1993) reported a RR of 30 in a high incidence context. Thus, it appears reasonable to assume that contact surveillance, while arduous and time-consuming, could yield significant results in the control and elimination of leprosy in endemic areas.

However, caution must be taken when generalising the incidence data found in our study. The possibility of bias in selecting individuals for cohort studies must be acknowledged because the demand for health care services in a reference centre occurs spontaneously or by referral. Thus, the group of index patients were not randomly selected from the population with leprosy in the municipality. Consequently, perhaps reflecting the preferential referral of the most serious cases, there is a clear predominance of MB cases among index patients $(71.2 \%)$. Thus, our incidence rates may be higher than average.

One of the central objectives of the present study was to attempt to identify a subgroup of contacts at greater risk of acquiring leprosy (i.e., a subgroup representing a higher risk of illness).

The results of the logistic regression model indicate that the non-BCG-vaccinated contacts of patients with a MB clinical form of leprosy who had a negative initial Mitsuda test are a group at high risk of illness (Table). The initial Mitsuda test result reflects a variety of factors that are extremely important in evaluating an individual's susceptibility to leprosy. For example, these factors include an immune response to leprosy that is influenced by human leukocyte antigen (Neeloo et al. 1997) and the effectiveness of this response in producing gamma-interferon (Sampaio et al. 1991). In other words, although the Mitsuda reaction is a relatively crude test in which the results are influenced by multiple dynamic factors that change over time and function in a series of individual and clinical variables, the results appear to be an indicator of risk among household leprosy contacts, regardless of BCG vaccination or the primary patient's clinical form of leprosy.

In a series of studies, Fine (Fine 1988, Fine et al. 1994, 1997) demonstrated the effectiveness of BCG as a protective agent against leprosy. Among the studies from Fiocruz cohort, one case-control study (Alvim 1993) confirmed the protective role of BCG. Duppre 2008 and Duppre et al. (2008) found that the effectiveness of BCG vaccination is related to age; its protective effect is stronger during the first years of life. The study also demonstrated that contact re-vaccination (in contacts with a BCG scar) confers additional protection.

The third risk factor, having contact with a MB clinical form of leprosy, was expected because this clinical form indicates a high bacillary load (Noordeen 1993, van Beers et al. 1994). What the model appears to indicate, however, is that this factor alone cannot lead to leprosy disease; certain immunological and individual factors have as much or more importance in the development of the disease. It is unclear whether most or all of the household contacts of MB leprosy patients are infected, but do not develop the disease. Nonetheless, it is probable that the association between the MB clinical forms and illness shown in the model (Table) is related to the fact that the infected contacts in this group have a higher index than the PB patient contacts and that this higher index is related to a greater probability of illness. Again, it is possible that confirming the existence of asymptomatic carriers will provide a better understanding of disease transmission and detecting these carriers may be one of the most useful strategies for controlling leprosy in the future, particularly among household contacts.

Finally, Sales et al. (2011) used a multi-level model to characterise the risk factors among contacts who acquired the disease and among co-prevalent contacts who were already diseased, but not yet diagnosed. This study adds to the evidence concerning the protective effect of BCG addressed in previous studies and it also showed the difference between the risk factors for co-prevalent cases vs. incident cases. Among the co-prevalent patients, socioeconomic risk factors are especially significant. Among the patients who acquired the disease (incident cases), the most prominent risk factors were related to a higher bacillary load caused by close exposure to leprosy patients.

Using computer modelling techniques (SIMCOLEP) to study a cohort of contacts in Bangladesh, Fischer et al. (2010) concluded that there is heterogeneity in contact susceptibility and that the household contact him/herself is a significant factor independent of other susceptibilities.

In summary, the results of all the studies on leprosy contact and the cumulative evidence presented in this review suggest an urgent need for continuous leprosy contact surveillance. Continuous surveillance is by far the best strategy to reduce the incidence rate of leprosy in the future. This strategy includes BCG vaccination, detection of anti-PGL-1 IgM and follow-up of newly treated

\section{TABLE}

Specification of the final model of logistical regression step by step

\begin{tabular}{lcccc}
\hline Variable & Coefficient & $\mathrm{p}$ & $\begin{array}{c}\text { Odds } \\
\text { ratio }\end{array}$ & $\begin{array}{c}\text { Interval of } \\
\text { reliance } \\
(95 \%)\end{array}$ \\
\hline Mitsuda & 1.129 & $<0.001$ & 3.093 & $1.735-5.514$ \\
BCG & -0.961 & $<0.001$ & 0.380 & $0.215-0.672$ \\
$\begin{array}{l}\text { Clinical form of } \\
\text { the primary case }\end{array}$ & 0.935 & 0.010 & 2.547 & $1.249-5.192$ \\
\hline
\end{tabular}

the variables included in the final model were the Mitsuda test, the vaccination by BCG and the clinical form of the primary case. The model was specified for the dependent variable leprosy and the intervals of reliance for each parameter estimated are presented. 
patients (particularly measuring the bacilloscopic index of these patients). Efforts to improve the tools used to diagnose infections, such as diagnostic tools that measure biomarkers, are welcome. These conclusions indicate the necessity of strengthening contact surveillance efforts in areas with high numbers of new leprosy cases, including some regions of Brazil.

\section{REFERENCES}

Abel L, Demenais F 1988. Detection of major genes for susceptibility to leprosy and its subtypes. Genet 42: 256-266.

Alvim MFS 1993. Efeito protetor do BCG na hanseníase. Um estudo caso-controle, MsD Thesis, Universidade do Estado do Rio de Janeiro, Rio de Janeiro, 140 pp.

Barreto JG, Guimarães LS, Leão MRN, Ferreira DVG, Lima RAA, Salgado CG 2011. Anti-PGL-1 seroepidemiology in leprosy cases: household contacts and school children from a hyperendemic municipality of the Brazilian Amazon. Lep Rev 82: 358-370.

Bobosha K, van Schip JJC, Esquenazi DA, Guimarães MM, Martins MV, Bekele Y, Fantahun Y, Aseffa A, Franken KLMC, Gismondi RC, Pessolani MCV, Ottenhoff THM, Pereira GMB, Geluk A 2012. Peptides derived from Mycobacterium leprae ML1601c. Discriminate between leprosy patients and healthy endemic controls. J Trop Med 2012: 132049.

Chanteau S, Glaziou P, Plichart C, Luquiaud P, Plichart R, Faucher JF, Cartel JL 1993. Low predictive value of PGL-I serology for the early diagnosis of leprosy in family contacts: results of a 10year prospective field study in French Polynesia. Int J Lepr Other Mycobact Dis 61: 533-541.

Duppre NC, Camacho LA, da Cunha SS, Struchiner CJ, Sales AM, Nery JAC, Sarno EN 2008. Effectiveness of BCG vaccination among leprosy contacts: a cohort study. Trans $R$ Soc Trop Med Hyg 102: 631-638.

Duppre NC 2008. Risco de desenvolver hanseníase em contatos de pacientes, segundo positividade ao teste anti-PGL-I e situação vacinal (BCG), PhD Thesis, Fundação Oswaldo Cruz, Rio de Janeiro, $229 \mathrm{pp}$.

Feenstra SG, Nahar Q, Pahan D, Richardus JH 2012. Social contact patterns and leprosy disease: a case-control study in Bangladesh. Epidemiol Infect 14: 1-9.

Feitosa MF, Borecki I, Krieger H, Beiguelman B, Rao DC 1995. The genetic epidemiology of leprosy in a Brazilian population. $A m J$ Hum Genet 6: 1179-1185.

Fischer EAJ, de Vlas SJ, Habbema JDF, Richardus JH 2011. The long term effect of current and new interventions on the new case detection of leprosy: a modeling study. PLoS Negl Trop Dis 5: e1330.

Fine PEM 1988. BCG vaccination against tuberculosis and leprosy. British Medical Bulletin 44: 691-703.

Fine PEM 1982. Leprosy: the epidemiology of a slow bacterium. Epidemiol Rev 4: 161-168.

Fine PEM, Sterne JAC, Pönnighaus JM, Bliss L, Saul J, Chihana A, Munthali M, Warndorff DK 1997. Household and dwelling contact as risk factors for leprosy in northern Malawi. Am J Epid 146: $91-102$.

Fine PEM, Sterne JAC, Pönnighaus JM, Rees RJW 1994. Delayedtype hypersensitivity, mycobacterial vaccines and protective immunity. The Lancet 344: 1245-1249.

Fischer E, de Vlas S, Meima A, Habbema D, Richardus J 2010. Different mechanisms for heterogeneity in leprosy susceptibility can explain disease clustering within households. PLOS ONE 5: e14061.

Goulart IMB, Souza DOB, Marques CR, Pimenta, VL, Gonçalves MA, Goulart LR 2008. Risk and protective factors for leprosy development determined by epidemiological surveillance of household contacts. Clin Vac Immunol 15: 101-105.

Lazaro FP, Werneck RI, Mackert CCO, Cobat A, Prevedello C, Pimentel RP, Macedo GMM, Eleutério AM, Vilar G, Abel L, Xavier MB, Alcais A, Mira MT 2010. A major gene controls leprosy susceptibility in a hyperendemic isolated population from North of Brazil. J Infec Diseases 201: 1598-1605.

Matos HJ, Duppre N, Alvim MF, Machado-Vieira LM, Sarno EN, Struchiner CJ 1999. Leprosy epidemiology in a cohort of household contacts in Rio de Janeiro (1987-1991). Cad Saude Publica 15: $533-542$.

Moet FJ, Pahan D, Schuring RP, Oskam L, Richardus JH 2006. Physical distance, genetic relationship, age and leprosy classification are independent risk factors for leprosy in contacts of patients with leprosy. J Infect Dis 193: 346.

Moura RS, Calado KL, Oliveira MLW, Bührer-Sékula S 2008. Sorologia da hanseníase utilizando PGL-1: revisão sistemática. Rev Soc Bra Med Trop 41 (Suppl. II): 11-18.

Neeloo S, Agrawal S, Rastogi AK 1997. Infectious disease and immunity: special reference to major histocompatibility complex. Emerg Infect Dis 3: 41-49.

Noordeen SK 1993. Leprosy 1962-1992. Epidemiology and control of leprosy - a review of progress over the last 30 years. Trans $R$ Soc Trop Med Hyg 87: 515-517.

Ranade MG, Joshi GY 1995. Long-term follow-up of families in an endemic area. Indian J Lepr 67: 411-425.

Richardus JH, Habbema JD 2007. The impact of leprosy control on the transmission of M. leprae: is elimination being attained? Lepr Rev 78: 330-337.

Ridley DS, Jopling WH 1962. A classification of leprosy for research purposes. Lepr Rev 33: 119-128.

Ridley DS, Jopling WH 1966. Classification of leprosy according to immunity. Int J Lepr Other Mycobact Dis 34: 255-273.

Rodriguez G, Orozco LC 1996. Lepra, Instituto Nacional de Salud, Santafé de Bogotá, 220 pp.

Saad MHF, Medeiros MA, Gallo MEN, Gontijo PP, Fonseca LS 1990. IgM immunoglobulins reacting with the phenolic glycolipid-1 antigen from Mycobacterium leprae in sera of leprosy patients and their contacts. Mem Inst Oswaldo Cruz 85: 191-194.

Sales AM, Ponce de Leon A, Duppre NC, Hacker MA, Nery JAC 2011. Leprosy among patient contacts: a multilevel study of risk factors. PLoS Negl Trop Dis 5: e1013.

Sampaio EP, Moreira AL, Kaplan G, Alvim MFS, Duppre NC, Miranda CF, Sarno EN 1991. Mycobacterium leprae-induced interferon- $\gamma$ production by household contacts of leprosy patients: association with development of active disease. J Infec Dis 164: 990-993.

Shen JP, Zhang GC, Chen XS, Zhou M, Yu MW, Yan LB 2008. A long-term evolution on the epidemiological characteristics of leprosy, towards the goal of its elimination in 1949-2007 in China. Chin J Epidemiol 29: 1095-1100.

van Beers SM, Izumi S, Madjid B, Maeda Y, Day R, Klaster PR 1994. An epidemiological study of leprosy infection by serology and polymerase chain reaction. Int J Lepr Other Mycobact Dis 62: 1-9.

Vanderborght PR, Matos HJ, Salles AM, Vasconcellos SE, Silva-Filho VF, Huizinga TW, Ottenhoff TH, Sampaio EP, Sarno EN, Santos AR, Moraes MO 2004. Single nucleotide polymorphisms (SNPs) at -238 and -308 positions in the TNF-alpha promoter: clinical and bacteriological evaluation in leprosy. Int J Lepr Other Mycobact Dis 72: 143-148.

WHO - World Health Organization 1998. Elimination of leprosy as a public health problem (update). Weekly Epidemiol Rec 73: 169-176. 